hope, through this structural concept, that we can systematise our knowledge about the formation, stability and unique properties of the amorphous intertransition metal alloys and relate these properties to those of the crystalline counterparts.

\section{R. WANG}

Materials Department,

Pacific Northwest Laboratories,

Richland, Washington 99352

1. Finney J. L. Nature 266, 309-314 (1977),

2. Samson, S. in Developments in the Structural Chemistry of Alloy Phases (ed. Giessen, B. C.) 65-106 (Plenum, New York, 1969).

\section{Circular hydrogen bonds}

SAENGER ${ }^{1}$ rightly stresses both cooperativity and large deviations from tetrahedrality in water-involved hydrogen-bonding networks. Similar lattice unconstrained structures were observed previously when examining the hydration of biological macromolecules, especially proteins ${ }^{2-4}$, although the $\alpha$-cyclodextrin data examined by Saenger is of significantly better quality, and therefore more quantitative.

However, although I agree strongly that cooperative effects are relevant in $\mathrm{OH}$ group hydrogen bonding ${ }^{3-7}$, the existence of rings-be they homodromic or heterodromic-does not of itself conclusively indicate that cooperative effects are operating. Given even a purely pair-additive potential with an average coordination of more than two, we would expect ring structures to be a topological consequence. Current ideas on the structures of trigonal ${ }^{8}$ (for example, $\mathrm{B}_{2} \mathrm{O}_{3}$ ) and tetrahedral ${ }^{9}$ (for example $\mathrm{SiO}_{2}$ ) glasses necessarily involve rings without any known cooperativity. As in aqueous systems, simulation calculations yield extensive ring structures using purely pair-additive potentials ${ }^{10}$. The wide glassforming ability of the silicate glasses, and the many crystalline forms of silicates, underline the structural versatility of tetrahedral coordination; the ring structures are a consequence of the (noncooperative) interaction potential and the need to fill space within the constraints of the potential.

The $\alpha$-cyclodextrin crystal data are valuable for the quantification of deviations from ideal tetrahedrality, an effect previously noted with respect to poorer quality protein data ${ }^{4.11}$. The conventional wisdom of tetrahedrality in water is based on (1) the geometry of $\mathrm{sp}^{3}$ hybridisation; and (2) the structures of the ices. The ice structures are, however, subject to the lattice constraint; extrapolation to small disordered clusters of molecules is dangerous. Concerning (1), most recent large basis set $a b$ initio calculations on the water molecules ${ }^{12}$ show the lone pairs poorly separated; the deviation from tetrahedrality is large. This is backed up by neutron diffraction studies of hydrates, which show the directional control of the lone pairs to be relatively poor ${ }^{7.13}$. Strong tetrahedrality is also inconsistent with recent neutron scattering data on liquid water (J. C. Dore and I. P. Gibson, in preparation; refs 14,15 ); pair correlation functions predicted by non-cooperative tetrahedral models of the water molecule show far more short range tetrahedral structure than is observed ${ }^{15}$. In contrast, a cooperative potential which makes no such geometrical assumptions ${ }^{5.6}$ is in agreement with the data (P. Barnes, J. L. F., J. E. Quinn and J. D. Nicholas, in preparation).

Cooperativity and imperfect tetrahedrality are indeed important aspects of aqueous systems which are normally ignored ${ }^{3-7}$. Saenger's data give quantitative support to imperfect tetrahedrality, but does not of itself conclusively indicate that cooperativity is operating.

\section{J. L. FINNEY}

Department of Crystallography,

Birkbeck College, University of London, Malet Street, London, WC1, UK

1. Saenger, W. Nature 279, 343-344 (1979).

2. Berendsen. H. J. C. in Water: A Comprehensice Treatise. Vol. 5 red. Franks, F.) 293-330 (Plenum. New York. 1975).

3. Finney, J. L., Phil. Trans. R. Soc, B278. 3-32 (1977),

4. Finney, J. L. in Water: A Comprehensice Treatise Vol. 6 (ed. Franks, F.) (Plenum. New York, in the press).

5. Barnes, P. in Progress in Liquid Phisics led. Croxton. C A.) 381-428 (Wiley. Chichester, 1978)

6. Barnes, P., Finney, J. L.. Nicholas, J. D.. Quinn. J. \& Westerman. A. V. Acta crystallogr. A 34, Suppl.. S200 (1978;.

7. Finney, J. L., Farad. Disc chem. Soc. 66 in the press

8. Mozzi, R. L. \& Warren, B. E. J. appl. Crvstallogr. 3. 251-257 (1970).

9. Bell, R. J. \& Dean, P. Nature 212, 1354-1356 (1966)

10. Rahman. A. \& Stillinger, F. H. J. Am. chem. Soc. 95 , 7943-7948 (1973).

1. Finney, J. L. J. molec. Biol. 119, 415-44) (1978).

12. Diercksen, G. H. F. Theor, chim. Acta (Berl.) 21, 335-367 11971 .

13. Olovsson, I. \& Jonsson. P.G. in The Hydrogen Bond Vol. 2 leds Schuster, P., Zundel, G. \& Sandorfy, C.) 393-456 leds Schuster, P.. Zundel, G. \& Sand

4. Gibson, I. P thesis. Univ. Kent (1978).

15. Dore, J. C. Farad. Disc. chem. Soc. 66, tin the press).

SAENGER REPLIES-From a topological point of view, it is certainly justified to assume that water molecules and hydroxyl groups could form circular H-bonded structures without invoking the cooperative effect. The quadrupole functionality of water in combination with purely pairadditive interaction potentials could be sufficient to arrange water molecules into circular aggregates, similar to the formation of circles in $\mathrm{SiO}_{2}$ or $\mathrm{B}_{2} \mathrm{O}_{3}$ glasses, as pointed out by Finney. However, we should be aware that in $\mathrm{Si}-\mathrm{O}-\mathrm{Si}$ and $\mathrm{B}-\mathrm{O}-\mathrm{B}$ bonds the directions of the bonds (from donor to acceptor) are predetermined because $O$ is the electron acceptor and $\mathrm{Si}$ or $\mathrm{B}$ are the donors. It is one of the special properties of $\mathrm{H}$-bonds between hydroxyl groups $\mathrm{O}-\mathrm{H}$... O that both oxygen atoms can function either as donor or as acceptor. This duality is clearly resolved if $\mathrm{H}$-atoms are located and, because the direction of the $\mathrm{H}$-bonds is then known, the existence or absence of cooperativity between several such $\mathrm{H}$ bonds is directly evident.

In the absence of a cooperative effect, we should expect near-random directions of $\mathrm{O}-\mathrm{H} \cdots \mathrm{O}$ hydrogen bonds, be they in endless chains or in circular structures (which then would be heterodromic). Because the neutron data on $x$-cyclodextrin hexahydrate have enabled us to locate all $\mathrm{H}$-atoms unambiguously, the directions of all the $\mathrm{H}$-bonds are known. We find that in the two chain-like structures as well as in the homo- and antidromic circles all the $\mathrm{H}$-bonds are undirectional, a strong indication for the existence of the cooperative effect. Obviously, both topological and cooperative effects favour and stabilise circular $\mathrm{H}$-bonded structures.

Bogdan Lesyng (Warsaw University) has performed PCILO calculations on chain-like and circular $\mathrm{H}$-bonds (in preparation). $\mathrm{He}$ compared H-bonding energies of these structures with those of the respective pairwise, isolated $\mathrm{O}-\mathrm{H} \cdots \mathrm{O}$ interactions. The influence of the cooperative effect is marginal, about $1 \%$ of the total $\mathrm{H}$-bonding energy, in the two antidromic circles, but $7.3 \%$ in the homodromic circle and $9.8 \%$ in the chainlike structure. The data suggest that cooperativity does exist in these $\mathrm{H}$ bonded structures but its magnitude depends strongly on the arrangement of the H-bonds, one of the bonds in the antidromic circle III even being bifurcated. Another interesting result of these calculations concerns the dipole moments, $2.73 D$ for the homodromic circle but $\geqq 6$ $D$ for the antidromic circles and chain-like structure, suggesting that in water clusters, homodromic circles should be the preferred species.

\section{WOLFRAM SAENGER}

\author{
Abteilung Chemie, \\ Max-Planck-Institut \\ für experimentelle Medizin, \\ D-3400 Göttingen, FRG
}

\section{Matters Arising}

Matters Arising is meant as a vehicle for comment and discussion about papers that appear in Nature. The originator of a Matters Arising contribution should initially send his manuscript to the author of the original paper and both parties should, wherever possible, agree on what is to be submitted. Neither contribution nor reply (if one is necessary) should be longer than 300 words and the briefest of replies, to the effect that a point is taken, should be considered. 